\title{
Real-time density matrix renormalization group dynamics of spin and charge transport in push-pull polyenes and related systems
}

\author{
Tirthankar Dutta ${ }^{1,2, *}$ and S. Ramasesha, \\ ${ }^{1}$ Solid State and Structural Chemistry Unit, Indian Institute of Science, Bangalore 560012, India \\ ${ }^{2}$ Condensed Matter Theory Unit, Jawaharlal Nehru Centre for \\ Advanced Scientific Research, Jakkur Post, Bangalore 560064, India.
}

\begin{abstract}
In this paper we investigate the effect of terminal substituents on the dynamics of spin and charge transport in donor-acceptor substituted polyenes $\left(D-(C H)_{x}-A\right)$ chains, also known as push-pull polyenes. We employ a long-range correlated model Hamiltonian for the $D-(C H)_{x}-A$ system, and time-dependent density matrix renormalization group technique for time propagating the wave packet obtained by injecting a hole at a terminal site, in the ground state of the system. Our studies reveal that the end groups do not affect spin and charge velocities in any significant way, but change the amount of charge transported. We have compared these push-pull systems with donor-acceptor substituted polymethine imine (PMI), $D-(C H N)_{x}-A$, systems in which besides electron affinities, the nature of $p_{z}$ orbitals in conjugation also alternate from site to site. We note that spin and charge dynamics in the PMIs are very different from that observed in the case of push-pull polyenes, and within the time scale of our studies, transport of spin and charge leads to the formation of a "quasi-static" state.
\end{abstract}

PACS numbers: 72.15.Nj, 72.80.Le, 71.10.Fd

\section{INTRODUCTION}

Conjugated organic materials have a variety of applications. They are already being used in organic light emitting diodes (OLEDS) and organic thin-film transistors $\stackrel{1-4}{-4}$ They are also considered to be potential candidates for single-molecule electronic and spintronic devices. The issue of spin and charge transport in $\pi$ conjugated organic systems, therefore, is of prime importance. Thus, it is both important and necessary to understand the role of chemical modifications (substituents) on the dynamics of spin and charge transport in these systems. Electrons in these molecular materials experience strong electron correlations by virtue of reduced dimensionality and these interactions are also long-ranged since the systems are semiconducting. Therefore, to study transport of charge and spin in these systems, it is necessary to have appropriate models as well as techniques. Dynamics in interacting systems can be studied either in the frequency or in the real-time domain. The dynamics of strongly interacting systems in the frequency domain has been possible due to the Lanczos and correction vector techniques ${ }^{5-\underline{8}}$ using both exact diagonalization method for small systems, and the density matrix renormalization group (DMRG) method for large systems.

Studying dynamics in the real-time domain throws a lot more light into the transport mechanism. Exact time dependent techniques are possible only for small interacting systems. The advent of the time-dependent density matrix renormalization group (td-DMRG) method has allowed the study of out-of-equilibrium dynamics in large low-dimensional strongly correlated systems $\stackrel{\underline{9}-11}{-11}$ Recently, we have investigated non-equilibrium dynamics of spin and charge transport in unsubstituted polyenes, which are a class of $\pi$-conjugated organic materials, using the double time window targeting (DTWT) td-DMRG technique developed by us: $\frac{12}{2}$ In the present paper we extend our studies to address non-equilibrium spin and charge dynamics in the push-pull polyenes, characterized by the presence of an electron donating (push) group, and an electron accepting (pull) group, schematically represented as $D-(\mathrm{CH})_{x}-A$. Presence of the donor and acceptor groups polarizes the $\pi$-electron bridge such that there is partial charge transfer from the donor to the acceptor group. The electronic structure of a pushpull polyene can be described by two canonical valence bond (VB) structures, namely, a neutral polyene structure and a zwitterionic structure, also known as charge transfer $(\mathrm{CT})$ configuration, where an electron is transferred from $D$ to $A \stackrel{13}{\underline{13}}$ This leads to the naive expectation that these groups will have significant influence on spin-charge dynamics. The push-pull polyenes have been so far studied mostly in the context of nonlinear optical response $\underline{\underline{13}} \underline{-15}$

In this paper we report our time-dependent DMRG studies on the spin and charge transport in push-pull polyenes and compare these results with those on polymethine imines which are quasi one-dimensional systems with alternate $\mathrm{C}$ and $\mathrm{N}$ atoms in the conjugation backbone. The organization of the paper is as follows: In the next section we provide details about the model Hamiltonian and the computational method used in this study. In Sec. III we present our results with discussions. Section IV concludes the paper. 


\section{MODEL AND COMPUTATIONAL METHODOLOGY}

The Pariser-Parr-Pople (PPP) Hamiltonian $\underline{16}$ with dimerization and site energies is appropriate for describing the low energy physics of $\pi$-conjugated systems. The PPP model Hamiltonian is given by

$$
\begin{aligned}
\hat{H}_{\mathrm{PPP}} & =\sum_{i=1}^{N-1} \sum_{\sigma} t_{0}\left[1-(-1)^{i} \delta\right]\left(\hat{c}_{i, \sigma}^{\dagger} \hat{c}_{i+1, \sigma}+\text { h.c. }\right) \\
& +\sum_{i=1}^{N} \epsilon_{i} \hat{n}_{i}+\sum_{i=1}^{N} \frac{U_{i}}{2} \hat{n}_{i}\left(\hat{n}_{i}-1\right) \\
& +\sum_{i>j} V_{i j}\left(\hat{n}_{i}-z_{i}\right)\left(\hat{n}_{j}-z_{j}\right) .
\end{aligned}
$$

Here, $N$ is the number of carbon atoms in the polyene chain, $\hat{c}_{i, \sigma}^{\dagger}\left(\hat{c}_{i, \sigma}\right)$ creates (annihilates) an electron with spin orientation $\sigma$ in the $p_{z}$ orbital of the $i^{\text {th }}$ carbon atom, $t_{0}$ is the average transfer integral and, $\delta(0 \leq \delta \leq 1)$ is the bond alternation parameter. The orbital energy and on-site Coulomb repulsion of the $p_{z}$ orbital on the $i^{\text {th }}$ carbon atom are given by $\epsilon_{i}$ and $U_{i}$, respectively and $\hat{n}_{i}$ is the number operator on the $i^{\text {th }}$ site. $V_{i j}$ is the intersite Coulomb repulsion between sites $i$ and $j$, and $z_{i}$ is the on-site chemical potential at the $i^{\text {th }}$ site. In case of unsubstituted polyene systems, $\underline{18} U_{i}=11.26 \mathrm{eV}, \epsilon_{i}$ $=0$ and $z_{i}=1$, for all sites, $t_{0}=-2.4 \mathrm{eV}$ and $\delta=$ 0.07 . The intersite interaction between electrons, $V_{i j}$, is interpolated according to the Ohno scheme, $\stackrel{17}{\Perp}$ between $U$ for $r=0$ and $\frac{e^{2}}{r}$ for $r \rightarrow \infty$ as,

$$
V_{i j}=14.397\left[\left(\frac{28.794}{U_{i}+U_{j}}\right)^{2}+r_{i j}^{2}\right]^{-1 / 2} .
$$

We have used single-bond length of $1.495 \AA$, doublebond length of $1.299 \AA$, and a bond angle of $120^{\circ}$ between successive bonds. These parameters have correctly predicted a whole range of properties of the low-lying states of conjugated systems in general and polyenes in particular $\stackrel{19-21}{2}$ When push and pull groups are introduced, we assume that only the energies of those $p_{z}$ orbitals to which the push and pull groups are attached, change due to inductive effect and all other parameters of the model remain unchanged. The donor group raises the orbital energy of the first carbon atom to which it is attached by $+\epsilon_{D}$, while the acceptor group lowers the orbital energy of the last carbon atom to which it is attached by $-\epsilon_{A}$, where $\epsilon_{D}$ and $\epsilon_{A}>0$. We assume that $\epsilon_{D}$ $=-\epsilon_{A}$ (symmetric push-pull polyenes). We have studied push-pull polyenes of 30 and 40 carbon atoms with terminal push and pull groups, and have varied the pushpull strength $|\epsilon|$. Although, presence of the push and pull groups destroys both the electron-hole and the inversion symmetry, the total spin invariance of the Hamiltonian, remains preserved.
For studying the dynamics of spin and charge transport, an initial wave packet $|\psi(0)\rangle$ is constructed by annihilating an up spin electron from the first site of a push-pull polyene of $N$ sites, in the ground state, $\left|\phi_{\mathrm{gs}}^{0}\right\rangle$,

$$
|\psi(0)\rangle=c_{1, \uparrow}\left|\phi_{\mathrm{gs}}^{0}\right\rangle .
$$

The wave packet $|\psi(0)\rangle$ evolves under the influence of the PPP Hamiltonian [Eq. (1)] following the time-dependent Schr" odinger equation and temporal dependence of site charge density $\left\langle n_{i}(t)\right\rangle$, and site spin density $\left\langle s_{i}^{z}(t)\right\rangle$, of this wave packet can be computed as,

$$
\begin{aligned}
& \left\langle n_{i}(t)\right\rangle=\left\langle\psi(t)\left|\left(n_{i, \sigma}+n_{i,-\sigma}\right)\right| \psi(t)\right\rangle, \\
& \left\langle s_{i}^{z}(t)\right\rangle=\frac{1}{2}\left\langle\psi(t)\left|\left(n_{i, \sigma}-n_{i,-\sigma}\right)\right| \psi(t)\right\rangle .
\end{aligned}
$$

Here, $|\psi(t)\rangle$ is the wave packet at time $t$.

Real-time dynamics of the initial wave packet is stud-

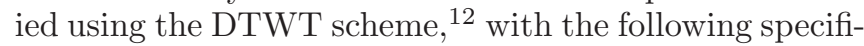
cations: number of density matrix eigenvectors (DMEVs) retained, $m=300$, time-step for evolution $\Delta \tau=0.0066$ $\mathrm{fs}$, total evolution time $T=33.0 \mathrm{fs}$, the number of time steps in each window is kept at 130 which corresponds to $0.858 \mathrm{fs}$, and the number of windows is 39 . Although we compute charge (spin) density at all the sites, we focus only on the quantities $\left\langle n_{1}(t)\right\rangle$ and $\left\langle n_{L}(t)\right\rangle$ and, $\left\langle s_{1}^{z}(t)\right\rangle$ and $\left\langle s_{L}^{z}(t)\right\rangle$ at the terminal sites attached to the substituents as these are sufficient for our purpose.

Another class of donor-acceptor substituted systems examined are the polymethine imines (PMI), with the molecular formula $D-(C H N)_{x}-A \underline{22} \underline{25}$ These systems have alternately donor $(\mathrm{C})$ and acceptor $(\mathrm{N})$ atoms in conjugation. The bonding in this polymer corresponds to $\cdots-C H=N-C H=N-\cdots$ and both carbon and nitrogen atoms are in $s p^{2}$ hybridization. This system has been studied extensively for linear and non-linear optical properties. The nitrogen $2 p_{z}$ orbitals are lower in energy than the carbon $2 p_{z}$ orbitals and intra-orbital repulsion of the nitrogen $2 p_{z}$ orbitals are greater than that of the carbon $2 p_{z}$ orbitals, both these can be rationalized on the basis of the more compact $2 p$ orbitals in nitrogen compared to carbon. The transfer integrals for $C=N$ and $C-N$ are $-2.767 \mathrm{eV}$ and $-2.317 \mathrm{eV}$, respectively; $C=N$ and $C-N$ bond lengths are $1.273 \AA$ And $1.425 \AA$; $U_{C}=11.26 \mathrm{eV}, U_{N}=12.34 \mathrm{eV} ; \epsilon_{C}=0.0 \mathrm{eV}, \epsilon_{N}=-2.96$ $\mathrm{eV}$. The initial wave packet is constructed as before [see Eq. (3)] and time evolved by the DTWT scheme using a smaller time-step of $\Delta \tau=0.0033$ fs, necessitated by the larger transfer integrals.

\section{RESULTS AND DISCUSSION}

In the absence of source and sink, the charge and spin of the hole decouple (spin-charge separation) and oscillate between sites 1 and $L$, with time. Hence, the time 


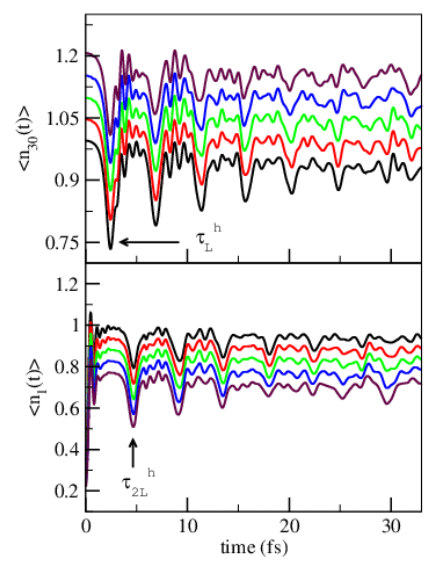

(III)

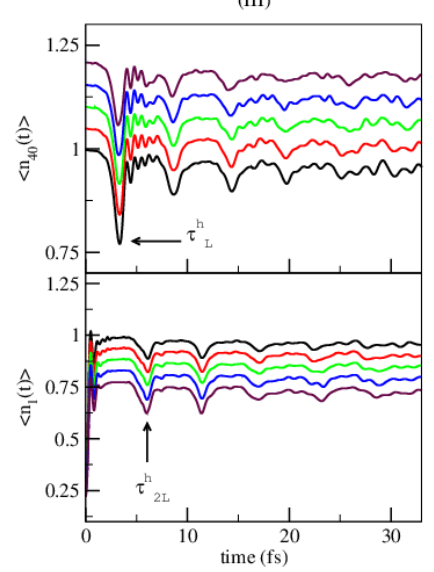

(II)

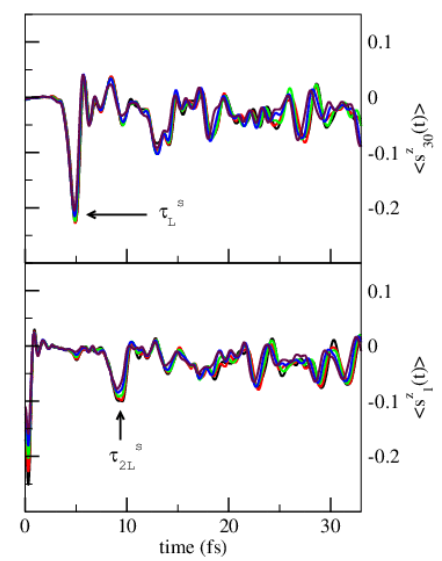

(IV)

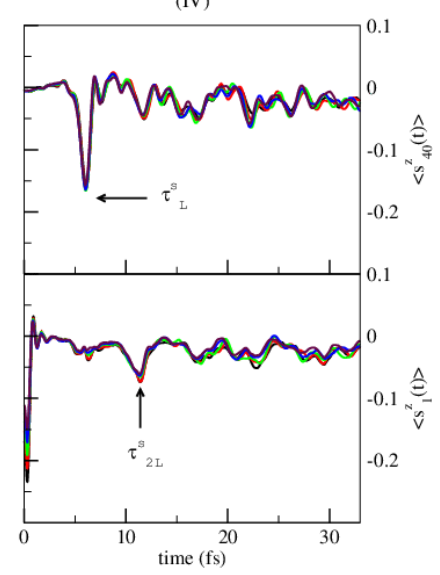

TABLE I: Variation in $\frac{\vartheta_{L}^{h}}{\vartheta_{F}}$ and $\frac{\vartheta_{L}^{s}}{\vartheta_{F}}$ in the dimerized $(\delta=0.07)$ Hubbard model with chain lengths 30 and 40, for $\frac{U}{|t|}=2.0$, 4.0 and 6.0. The same quantities for the $\delta=0.07$ PPP model is also quoted. $\mathrm{M}$ and $\mathrm{Q}$ stand for model and quantities, respectively.

\begin{tabular}{lccccccccc}
\hline \hline $\mathrm{Q}$ & $\frac{U}{|t|}=2.0$ & $\frac{U}{|t|}=4.0$ & $\frac{U}{|t|}=6.0$ & $\mathrm{PPP}$ \\
\hline$N$ & 30 & 40 & 30 & 40 & 30 & 40 & 30 & 40 \\
$\vartheta_{L}^{h} / \vartheta_{F}$ & 1.33 & 1.32 & 1.35 & 1.39 & 1.30 & 1.18 & 5.05 & 4.80 \\
$\vartheta_{L}^{s} / \vartheta_{F}$ & 0.97 & 0.91 & 0.69 & 0.80 & 0.68 & 0.80 & 2.49 & 2.63
\end{tabular}

in the time evolution profiles of $\left\langle n_{1}(t)\right\rangle$ and $\left\langle s_{1}^{z}(t)\right\rangle$. From these times, the charge and spin velocities of the injected hole are calculated as, $\vartheta_{L}^{s / h}=\frac{L}{\tau_{L}^{s / h}}$ and $\vartheta_{2 L}^{s / h}=\frac{2 L}{\tau_{L}^{s / h}}$.

The dynamics of spin and charge transport in systems with reduced dimensionality and electron-electron interactions, are expected to be different from each other, owing to spin-charge separation. When electron correlations are absent, the charge and spin velocities, $\vartheta_{L}^{h}$ and $\vartheta_{L}^{s}$, are equal to the Fermi velocity, $\vartheta_{F}$. From our earlier td-DMRG studies 26 we have found that the Fermi velocities of tight-binding chains of lengths 30 and 40 are 2.63 and $2.72(\AA / \mathrm{fs})$ respectively, for $\delta=0.0$, and 2.43 and $2.49(\AA / \mathrm{fs})$, respectively, for $\delta=0.07$. Table I presents a comparison of the ratio of $\vartheta_{L}^{h} / \vartheta_{F}$ and $\vartheta_{L}^{s} / \vartheta_{F}$, for Hubbard chains of 30 and 40 sites with different $\frac{U}{|t|}$ values and $\delta=0.07$. These quantities are also computed for dimerized PPP chains with 30 and 40 sites, with $|\epsilon|=$ 0.0, and compared with those of Hubbard chains. As evident from Table I, the values of the ratios of $\vartheta_{L}^{h} / \vartheta_{F}$ and $\vartheta_{L}^{s} / \vartheta_{F}$ are much higher in the PPP model compared to the Hubbard model, due to the presence of long-range electron-electron interactions.

As with unsubstituted polyenes, 12 in push-pull polyenes (Fig. 1) the spin propagates slower than the charge with $\tau_{L / 2 L}^{s}>\tau_{L / 2 L}^{h}\left(\vartheta_{L / 2 L}^{s}<\vartheta_{L / 2 L}^{h}\right)$. However, we find that the $D$ and $A$ groups have no significant effect on spin and charge velocities in the push-pull polyenes. Consequently, the charge and spin velocities in push-pull polyenes remain almost equal to those in unsubstituted polyenes (Table II). The push-pull substitution however, has an effect on the amount of charge transported, as seen from the depths of the minima in the time evolution profiles of $\left\langle n_{1}(t)\right\rangle$ and $\left\langle n_{L}(t)\right\rangle$. However, depth of the minima in $\left\langle s_{1}^{z}(t)\right\rangle$ or $\left\langle s_{L}^{z}(t)\right\rangle$ do not change (see Fig. 1), implying that the push-pull groups have no influence on the spin degree of freedom of the hole.

This implies that even though the spin and charge velocities in $D-(C H)_{x}-A$ polyenes remain largely unaffected by the donor-acceptor strengths, the amount of charge transferred in unit time decreases with increase 
TABLE II: Variation of $\tau_{L}^{h}$ and $\tau_{L}^{s}$ (fs) and, $\vartheta_{L}^{h}$ and $\vartheta_{L}^{s}(\AA / \mathrm{fs})$ with chain length $(L)$, in symmetric push-pull polyene chains of length, 30 and 40 sites, with different values of $|\epsilon|(\mathrm{eV})$. The PPP model parameters are, $t_{0}=-2.4 \mathrm{eV}, U=11.26 \mathrm{eV}$, and $\delta=0.07 ; L=1.397(N-1-\delta) \AA, N$ being the number of sites, and $O$ stands for Observables.

\begin{tabular}{ccccccc}
\hline \hline$N$ & $O$ & $|\epsilon|=0.0$ & $|\epsilon|=0.5$ & $|\epsilon|=1.0$ & $|\epsilon|=1.5$ & $|\epsilon|=2.0$ \\
\hline \multirow{4}{*}{30} & $\tau_{L}^{h}$ & 2.45 & 2.45 & 2.44 & 2.44 & 2.42 \\
& $\tau_{L}^{s}$ & 4.96 & 4.92 & 4.90 & 4.87 & 4.83 \\
& $\vartheta_{L}^{h}$ & 16.50 & 16.50 & 16.57 & 16.57 & 16.71 \\
& $\vartheta_{L}^{s}$ & 8.15 & 8.22 & 8.25 & 8.30 & 8.37 \\
& $\left(\vartheta_{L}^{h} / \vartheta_{L}^{s}\right)$ & 2.02 & 2.01 & 2.01 & 2.00 & 2.00 \\
& $\tau_{L}^{h}$ & 3.35 & 3.34 & 3.30 & 3.25 & 3.19 \\
40 & $\tau_{L}^{s}$ & 6.10 & 6.08 & 6.05 & 6.03 & 6.01 \\
& $\vartheta_{L}^{h}$ & 16.24 & 16.28 & 16.42 & 16.74 & 17.05 \\
& $\vartheta_{L}^{s}$ & 8.92 & 8.95 & 8.99 & 9.02 & 9.05 \\
& $\left(\vartheta_{L}^{h} / \vartheta_{L}^{s}\right)$ & 1.82 & 1.82 & 1.83 & 1.86 & 1.88 \\
\hline \hline
\end{tabular}

in $|\epsilon|$ as seen from the decrease in depth of the minima, when a hole is doped at the donor site. We can expect the opposite of when we dope an electron at the donor site; more charge will be transported from the donor in these cases as $|\epsilon|$ increases. Our results indicate that push-pull substitutions do not alter the many-body character of the $D-(C H)_{x}-A$ polyenes, and that spin and charge velocities are controlled by the correlation strength while the magnitude of charge transfer is controlled by the strength of the substituents.

To understand in detail, the reason for the inability of the donor and acceptor groups to alter the many-body character of the $D-(C H)_{x}-A$ polyenes, we compute the inverse of the charge gap $\left(\Delta E_{c}\right)$ and spin gap $\left(\Delta E_{s}\right)$ of the push-pull polyenes. If $E_{g s}(N+1), E_{g s}(N-1)$ and $E_{g s}(N)$ denote the ground state energies of the $(N+1)$ particle, $(N-1)$-particle and $N$-particle systems respectively, then the charge gap is given by,

$$
\Delta E_{c}=E_{g s}(N+1)+E_{g s}(N-1)-2 E_{g s}(N) .
$$

Similarly, the spin gap is defined as,

$$
\Delta E_{s}=E_{0}\left(S_{\text {tot }}^{z}=3 / 2\right)-E_{0}\left(S_{\text {tot }}^{z}=1 / 2\right)
$$

where, $E_{0}\left(S_{\text {tot }}^{z}=3 / 2\right)$ and $E_{0}\left(S_{\text {tot }}^{z}=1 / 2\right)$ are the lowest energy states of the $D-(\mathrm{CH})_{x}-A$ polyenes with $S_{\text {tot }}^{z}$ $3 / 2$ and $1 / 2$, respectively. In Fig. 2, we have plotted the variation in $\tau_{L}^{h / s}$ and $1 / \Delta E_{c / s}$ as a function of the strength of the push-pull groups. It is clearly observed that the donor-acceptor groups fail to alter either of the gaps, as a result of which, the velocities of the spin and charge remain unaffected.

Comparing Figs. 1 and 3 it is clear that the dynamics of spin and charge transport in the $D-(C H N)_{x}-$ $A$ chains is very different from that in the push-pull polyenes, owing to the different $U$ values and site energies of carbon and nitrogen atoms. For example, unlike
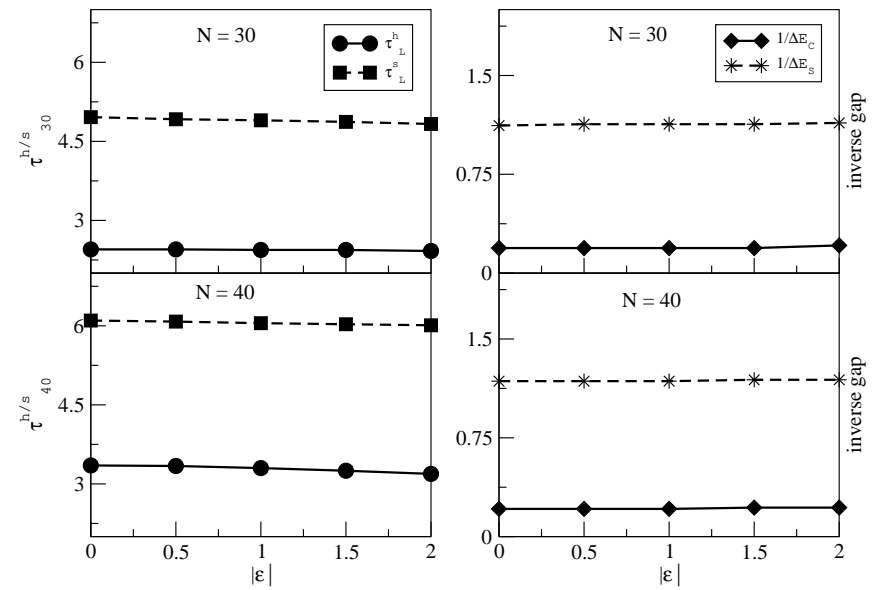

FIG. 2: Variation in $\tau_{L}^{h / s}$ (fs), left-hand top and bottom plots, and $1 / \Delta E_{c / s}\left(\mathrm{eV}^{-1}\right)$, right-hand top and bottom plots, as a function of strength of push-pull groups $(|\epsilon|=0.0,0.5,1.0$, 1.5 and 2.0), for polyene chains of 30 and 40 sites.

in push-pull polyenes (where the charge and spin of the injected hole after decoupling, oscillate back and forth between sites 1 and $L)$ in the $D-(C H N)_{x}-A$ systems, the oscillations are damped and the spin and charge of the injected hole do not return after reaching the chain end. This is demonstrated by the observation that, although the first dip in the time evolution profiles of $\left\langle n_{L}(t)\right\rangle$ and $\left\langle s_{L}^{z}(t)\right\rangle$ are well defined, there are no well defined second (major) minima in the $\left\langle n_{1}(t)\right\rangle$ and $\left\langle s_{1}^{z}(t)\right\rangle$ curves as a function of time. This may be because the hopping of an electron between unlike atoms is not a degenerate resonant tunneling process and hence, is not strictly reversible.

In order to investigate the fate of the injected hole, we study the temporal variation of total charge and spin densities in the left and right halves of PMI chains of 30 and 40 sites, for $|\epsilon|=0.0$ (Fig. 4). The total charge and spin densities for the left (right) half are defined as, $\left\langle N_{L(R)}(t)\right\rangle=\sum_{j \in L(R)}\left\langle n_{j}(t)\right\rangle$, and $\left\langle S_{L(R)}^{z}(t)\right\rangle=\sum_{j \in L(R)}$ $\left\langle s_{j}^{z}(t)\right\rangle(L \in[1, N / 2], R \in[N / 2+1, N])$, respectively. These quantities serve as simple but effective probes to understand the motion of charge and spin of the hole, as they travel from the half of the system where charge injection occured to the opposite half of the system. If the time evolution profiles of these observables show significant oscillatory behavior, it implies that the charge and spin propagate back and forth between the ends of the system. However, if the oscillatory behavior is not pronounced and both the observables attain some average value, it signifies that the charge (spin) moves in a such a manner that a "quasi-static" state is generated in which charge (spin) distribution of the system remains unchanged within the time of study. It is observed from Fig. 4 that for both the chain lengths, $\left\langle S_{R}^{z}(t)\right\rangle$ goes from 0.0 to -0.25 while, $\left\langle S_{L}^{z}(t)\right\rangle$ goes from an initial value of 
(I)

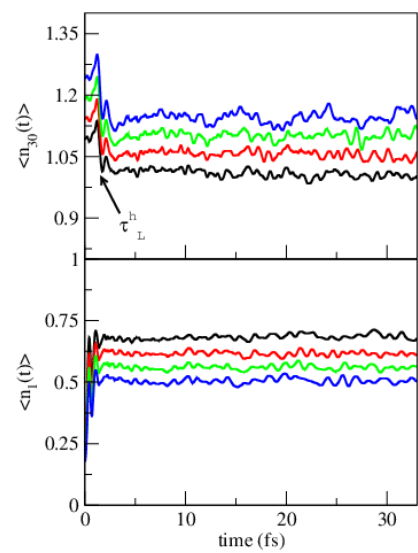

(III)

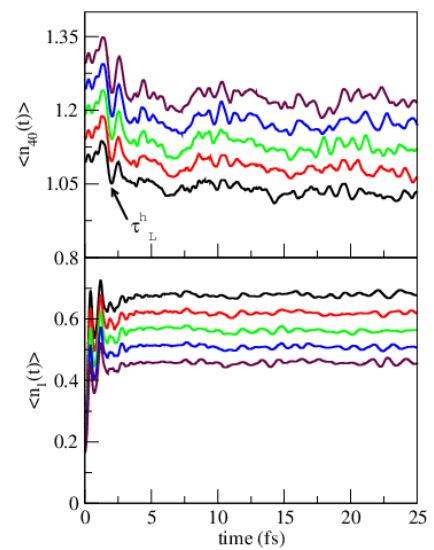

(II)

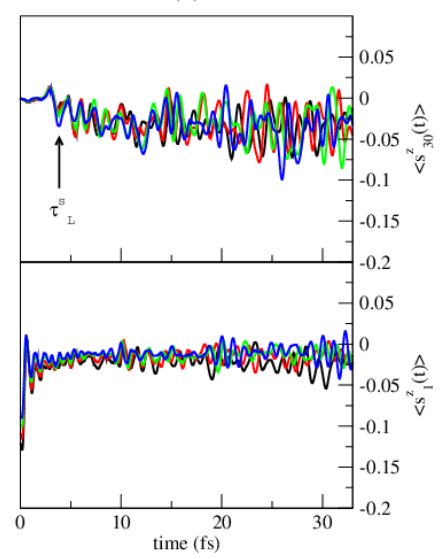

(IV)

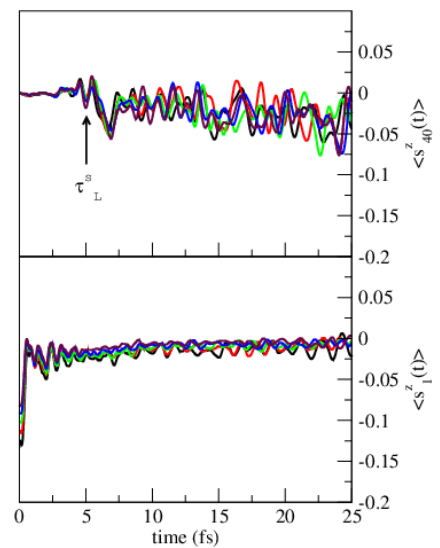

FIG. 3: (color online) Temporal variation in $\left\langle n_{i}(t)\right\rangle$ and $\left\langle s_{i}^{z}(t)\right\rangle$ for polymethine imine system. Panels (I) and (II) are for $N=30$ chains while (III) and (IV) are for $N=40$ chains. Panels (I) and (III) give evolution of charge while (II) and (IV) give spin density evolution. In each panel, bottom box gives the evolution of site 1 and the top box, evolution at the end of the chain $L$. In the charge density panels $((\mathbf{I})$ and (III)) $|\epsilon|$ is $0.0 \mathrm{eV}, 0.5 \mathrm{eV}, 1.0 \mathrm{eV}, 1.5 \mathrm{eV}$ and $2.0 \mathrm{eV}$ for curves from top to bottom in the $\left\langle n_{1}(t)\right\rangle$ boxes, and from bottom to top in the $\left\langle n_{L}(t)\right\rangle$ boxes. The same is the case in the spin density panels ((II) and (IV)). In color, $|\epsilon|=0.0$ $\mathrm{eV}$ (black), $0.5 \mathrm{eV}$ (red), $1.0 \mathrm{eV}$ (green), $1.5 \mathrm{eV}$ (blue), $2.0 \mathrm{eV}$ (maroon) curves. In top boxes of the panels (I), (II), (III) and (IV), $\tau_{L}^{h}$ and $\tau_{L}^{s}$ have been indicated by black arrow.

-0.5 to -0.25 . Once these quantities have attained the value of -0.25 , they start to oscillate with time about this average value. The charge densities for the left and right halves of the PMI chains are however, found to behave little differently. In the case of the 30 site chain it is observed that, $\left\langle N_{L}(t)\right\rangle$ and $\left\langle N_{R}(t)\right\rangle$ take the average values of $\sim 14.25$ and $\sim 14.75$, while for the longer chain of 40 sites, these quantities oscillate about a mean value of $\sim 19.5$. These data suggest that in the $(C H N)_{x}$ systems, with increase in system size, within the computational time a "quasi-static" state is achieved more rapidly.
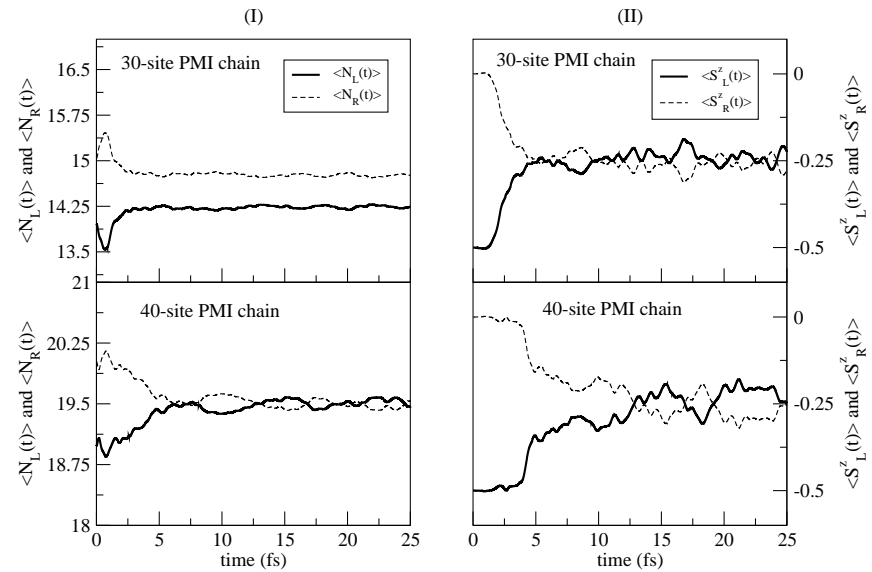

FIG. 4: Variation in $\left\langle N_{L(R)}(t)\right\rangle$ (plots in left box marked as (I)) and $\left\langle S_{L(R)}^{z}(t)\right\rangle$ (plots in right box marked as (II)) with time, for unsubstituted PMI chains $(|\epsilon|=0.0)$ of 30 and 40 sites. Solid curves correspond to $\left\langle N_{L}(t)\right\rangle$ and $\left\langle S_{L}^{z}(t)\right\rangle$, respectively, and dashed curves correspond to $\left\langle N_{R}(t)\right\rangle$ and $\left\langle S_{R}^{z}(t)\right\rangle$.

To understand this "quasi-static" state in more detail, we plot the time evolution profiles of charge and spin densities on carbon and nitrogen atoms in both halves of unsubstituted PMI chains of 30 and 40 sites (Fig. 5). These quantities are defined as,

$$
\begin{aligned}
\left\langle N_{L, C}(t)\right\rangle & =\sum_{j \in C}\left\langle n_{j}(t)\right\rangle ; \quad\left\langle S_{L, C}^{z}(t)\right\rangle=\sum_{j \in C}\left\langle s_{j}^{z}(t)\right\rangle, \\
\left\langle N_{R, C}(t)\right\rangle & =\sum_{j \in C}\left\langle n_{j}(t)\right\rangle ; \quad\left\langle S_{R, C}^{z}(t)\right\rangle=\sum_{j \in C}\left\langle s_{j}^{z}(t)\right\rangle, \\
\left\langle N_{L, N}(t)\right\rangle & =\sum_{j \in N}\left\langle n_{j}(t)\right\rangle ; \quad\left\langle S_{L, N}^{z}(t)\right\rangle=\sum_{j \in N}\left\langle s_{j}^{z}(t)\right\rangle, \\
\left\langle N_{R, N}(t)\right\rangle & =\sum_{j \in N}\left\langle n_{j}(t)\right\rangle ; \quad\left\langle S_{R, N}^{z}(t)\right\rangle=\sum_{j \in N}\left\langle s_{j}^{z}(t)\right\rangle,
\end{aligned}
$$

where $L \in[1, N / 2]$ and $R \in[N / 2+1, N]$. It is observed from Figs. 5(II) and 5(IV) that the oscillations in time, of $\left\langle S_{L / R, C}^{z}(t)\right\rangle$ and $\left\langle S_{L / R, N}^{z}(t)\right\rangle$, are opposite in phase, signifying that spin densities on the carbon and nitrogen atoms (in both half) are antiferromagnetically coupled to each other. However, the total spin density carried by carbon atoms in both halves is found to be less than that carried by the nitrogen atoms. Furthermore it is seen that with time, both $\left\langle S_{L, C}^{z}(t)\right\rangle$ and $\left\langle S_{L, N}^{z}(t)\right\rangle$ decrease, while $\left\langle S_{R, C}^{z}(t)\right\rangle$ and $\left\langle S_{R, N}^{z}(t)\right\rangle$ increase in magnitude, keeping the total spin density in either half at -0.25. From Figs. 5(I) and 5(III) we see that the average value of $\left\langle N_{L / R, C}(t)\right\rangle$ is less than $\left\langle N_{L / R, N}(t)\right\rangle$, and the total charge density carried by nitrogen atoms on the right half of PMI chains is more compared to that in the left half in line with the fact that the nitrogens are the "acceptor" atoms, being more electronegative. The opposite is seen for the carbon atoms. The time evolution 

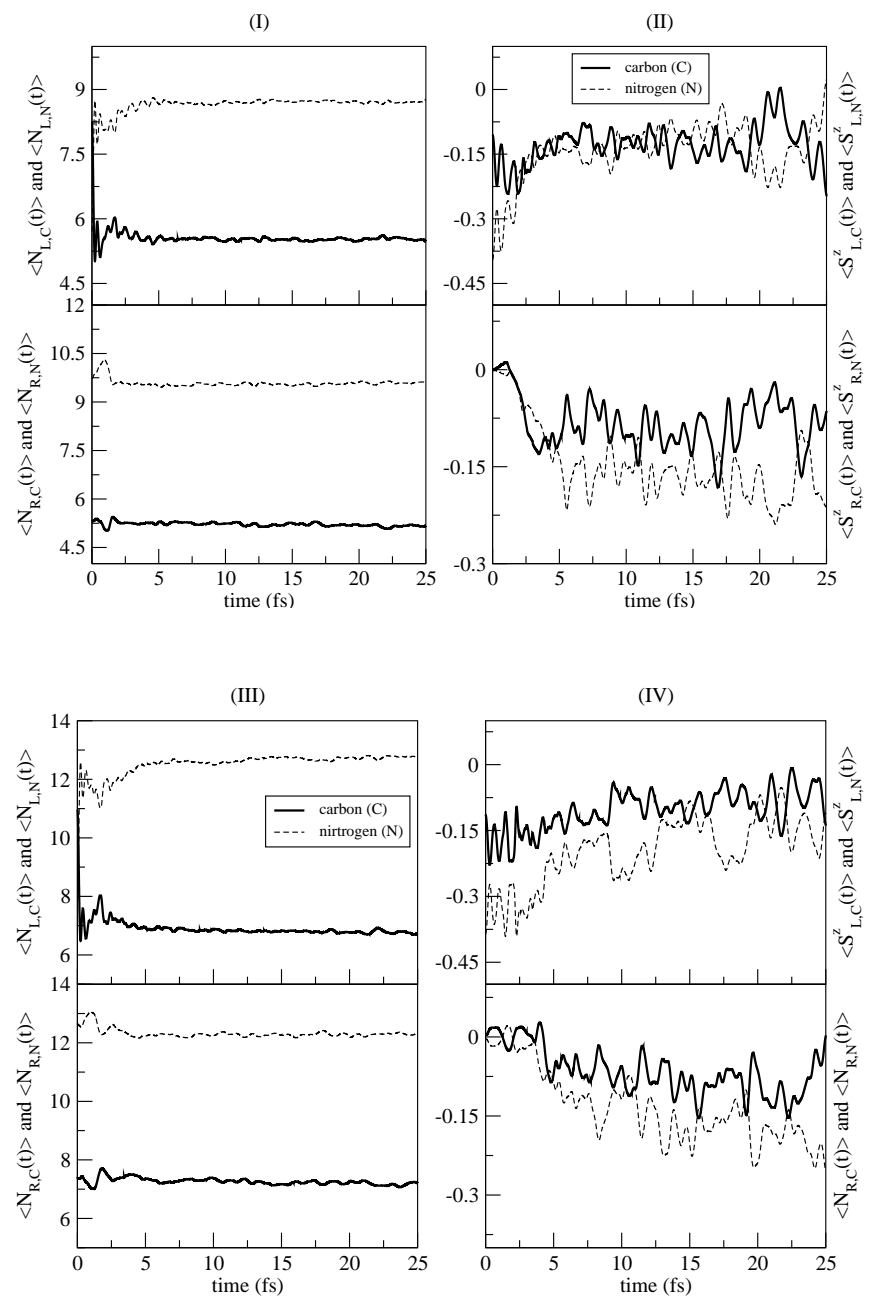

FIG. 5: Variation in total charge and spin densities of carbon and nitrogen atoms, with time, in the left and right halves of unsubstituted PMI chains $(|\epsilon|=0.0)$ with 30 and 40 sites. Top and bottom boxes correspond to $N=30$ and 40 sites, respectively. Solid and dashed curves pertain to carbon and nitrogen, respectively. In the top box, plots marked (I) refer to charge density variation and plots marked (II) refer to spin density variation, in left (upper plots) and right (lower plots) half of the system, respectively. The same holds for plots marked as (III) and (IV), in the bottom box.

profiles of total spin densities on carbon and nitrogen in both halves, compared to the total charge densities, are found to be more oscillatory.

All these observations suggest that the "quasi-static" state is characterized by charge and spin of the hole distributed between the left and right halves of the chain in almost equal amount, the positive or hole charge predominantly residing on carbon atoms while the spin, on nitrogen atoms. The $N$ atoms being more electronegative than the $C$ atoms $\left(\epsilon_{N}=-2.96 \mathrm{eV}, \epsilon_{C}=0.0 \mathrm{eV}\right)$, prefer being electron rich by accommodating more charge density. However, due to large on-site Coulomb repulsion
$\left(U_{N}=12.34 \mathrm{eV}\right)$, the average site charge on $N$ atoms is slightly larger than 1.0 which is observed from the charge density distribution in the neutral ground state and the initial state. On the other hand, due to $U_{C}=$ $11.26 \mathrm{eV}$ and $\epsilon_{C}=0.0$, the carbon atoms are slightly electron deficient in the $(C H N)_{x}$ system. Thus, propagation of the charge and spin degrees of freedom of the hole in this polarized background results in formation of the "quasi-static" state, which once formed, prevents both the charge and spin from returning to the site of injection as it requires reversal of polarization and is hence, energetically unfavorable.

In unsubstituted PMI chains, $\tau_{L}^{h}$ and $\tau_{L}^{s}$ are $1.75 \mathrm{fs}$ and 3.65 fs for $N=30$, and 2.01 fs and 6.60 fs for $N$ $=40$. We find that the $\vartheta_{h} / \vartheta_{s}$ ratios for the $N=30$ is 2.08 and for $N=40,3.29$. The $\vartheta_{h} / \vartheta_{s}$ ratio for a PMI chain of 30 sites agrees well with that of push-pull polyene with $N=30(|\epsilon|=0)$. However, this ratio for $N$ $=40(|\epsilon|=0)$ is much higher in the PMI chain compared to push-pull polyene of same size. This is opposite to the behavior exhibited by push-pull polyenes, where the $\vartheta_{h} / \vartheta_{s}$ ratio decreases with increasing chain length. In both polyenes and polymethine imines, the $\vartheta_{h} / \vartheta_{s}$ ratios do not saturate, indicating that the $\pi$-coherence lengths for transport are rather long. It also appears that alternate donor-acceptor sites along the chain enhance the velocities of both charge and spin. We have also studied substituents at the terminal sites in the $(C H N)_{x}$ systems and as with the polyenes, the strength of the push-pull groups do not affect the charge and spin velocities, or the nature of the "quasi-static" state.

\section{CONCLUSIONS}

To conclude, our studies show that the velocities of charge and spin transport are not affected by push-pull substituents, although the magnitude of charge transport depends on the push-pull strength. In the related $(C H N)_{x}$ system we find that, due to the underlying polarized structure both the spin and charge transport are affected, and charge travels much faster than spin compared to push-pull systems. Furthermore, the transport of charge and spin results in the formation of a "quasistatic" state in which the injected hole resides in both halves of the system, almost in equal amount. We also note that in the PMIs the finite size effects are very large compared to the push-pull polyenes, suggesting much longer $\pi$-coherence lengths.

\section{ACKNOWLEDGMENTS}

This work was supported by DST India and the Swedish Research Link Program under the Swedish Research Council. 
* Electronic address: tirthankar@sscu.iisc.ernet.in

$\dagger$ Electronic address: ramasesh@sscu.iisc.ernet.in

1 A. Dodabalapur, L. Torsi, and H. E. Katz, Science, 268, 270 (1995).

2 A. Dodabalapur, H. E. Katz, L. Torsi, and R. C. Haddon, Science, 269, 1560 (1995).

3 J. H. Burroughes, D. D. C. Bradeley, A. R. Brown, R. N. Marks, K. Machey, R. H. Friend, P. L. Burns, and A. B. Holmes, Nature (London), 347, 539 (1990).

4 A. Nitzan, and M. A. Ratner, Science, 300, 1384 (2003).

${ }^{5}$ K. Hallberg, Phys. Rev. B 52, R9827 (1995).

6 S. Ramasesha and Z. G. Soos, Chem. Phys. Lett. 153, 171 (1988); S. Ramasesha, S. K. Pati, H. R. Krishnamurthy, Z. Shuai and J. L. Brédas, Syn. Met. 85, 1019 (1997).

7 E. Jeckelmann, F. Gebhard, and F. H. L. Essler, Phys. Rev. Lett. 85, 3910 (2000); E. Jeckelmann, Phys. Rev. B 66, 045114 (2002).

8 T. D. Kühner and S. R. White, Phys. Rev. B 60, 335 (1999).

9 H. G. Luo, T.Xiang, and X. Q. Wang, Phys. Rev. Lett. 91, 049701 (2003).

10 A. E. Feiguin and S. R. White, Phys. Rev. Lett 93, 076401 (2004); A. J. Daley, C. Kollath, U. Schollwöck, and G. Vidal, J. Stat. Mech.: Theor. Exp. P04005 (2004); A. E. Feiguin and S. R. White, Phys. Rev. B 72, 020404(R) (2005).

11 U. Schollwöck, Rev. Mod. Phys. 77, 259 (2005).

12 T. Dutta and S. Ramasesha, Phys. Rev. B 82, 035115 (2010).

13 T. A. Skothem, R. L. Elsenvaumer, and J. R. Reynolds (eds): Handbook of Conducting Polymers, (Marcel Dekker, Inc., 1998). (see references therein)

14 L. T. Cheng, W. Tam, S. R. Marder, A. E. Stiegman, G. Rikken, and C. W. Sprangler, J. Phys. Chem., 95, 10643
(1991).

15 M. Blanchard-Desce, J. M. Lehn, M. Barzoukas, I. Ledoux, and J. Zyss, Chem. Phys., 181, 281 (1994); S. R. Marder, L.T. Cheng, B. G. Tiemann, A. C. Friedli, M. BlanchardDesce, J. W. Perry, and J. Skindhoj, Science, 263, 511 (1994); M. Blanchard-Desce, V. Bloy, J. M. Lehn, C. Runser, M. Barzoukas, A. Fort, and J. Zyss, Proc. SPIE bf 2143, 20 (1994); M. Blanchard-Besce, C. Runser, A. Fort, M. Barzoukas, J. M. Lehn, V. Bloy, and V. Alain, J. Chem. Phys. 199, 253 (1995).

16 R. Pariser and R. G. Parr, J. Chem. Phys. 21, 466 (1953); J. A. Pople, Trans. Farad. Soc. 49, 1375 (1953).

17 K. Ohno, Theor. Chem. Acta. 2, 219 (1964); G. Klopman, J. Am. Chem. Soc. 86, 4550(1964).

18 S. Ramasesha, Proc. Indian Acad. Sci. 96, 509 (1986); S. Ramasesha, J. Mol. Struc. 194, 149 (1989).

19 Z. G. Soos and S. Ramasesha, Phys. Rev B 29, 5410 (1984).

20 S. Ramasesha and Z. G. Soos, J. Chem. Phys. 80, 3278 (1984).

21 I. D. L. Albert and S. Ramasesha, J . Phys. Chem. 94, 6540 (1990).

22 A. Karpfen, Chem. Phys. Lett. 64, 299 (1979).

23 J. L. Bredas, B. Themans, and J. M. Andre, J. Chem. Phys. 78, 6137 (1983); D. Jacquemin, B. Champagne, and J. M. André, Synthetic Metals 80, 205 (1996); D. Jacquemina, B. Champagnea, J. M. André, and B. Kirtman, Chem. Phys. 213, 217 (1996); D. Jacquemin, E. A. Perpéte, and J. M. André, Int. J. Quan. Chem. 105, 553 (2005).

24 I. D. L. Albert, P. K. Das, and S. Ramasesha, Chem. Phys. Lett. 176, 217 (1991).

${ }^{25}$ G. Chen, Z. M. Su, Z. W. Shen, and Y. J. Yan, J. Chem. Phys. 109, 2565 (1998).

26 T. Dutta and S. Ramasesha, Comput. Lett. 3, 457 (2007). 